\section{IT'S A MALE WORLD: EL SESGO SEXUAL DE LOS MODELOS ANIMALES EN BIOLOGÍA}

\author{
Federico Bernabé Blach \\ Universidad Nacional de Quilmes \\ Consejo Nacional de Investigaciones Científicas y Técnicas (CONICET) \\ ORCID iD: https://orcid.org/0000-0002-4273-7786 \\ fnbernabeblach@gmail.com \\ Leandro Giri \\ Sociedad Argentina de Análisis Filosófico \\ Consejo Nacional de Investigaciones Cientificas y Técnicas (CONICET) \\ ORCID iD: https://orcid.org/0000-0002-7068-9750 \\ leandrogiri@gmail.com
}

Cómo citar este artículo/Citation: Bernabé Blach, F. y Giri, L. (2019). It's a Male World: el sesgo sexual de los modelos animales en biología. Arbor, 195 (791): a492. https://doi. org/10.3989/arbor.2019.791n1005

Recibido: 04 mayo 2017. Aceptado: 14 diciembre 2018.

RESUMEN: En el presente trabajo se analizan desde un punto de vista metateórico los modelos animales y su uso en la investigación en el ámbito de las ciencias de la vida. A partir de la evaluación de una nutrida literatura científica que denuncia un sesgo sexual en las prácticas de investigación que utilizan dichos modelos, se buscan los supuestos teóricos implícitos que les dan fundamento y se argumenta que lejos de suponer una torpeza metodológica forman parte de una extensa tradición ubicua en la historia de la biología. Por otra parte, se exponen los motivos teóricos para el reemplazo de los mencionados supuestos, a partir del análisis filosófico e histórico de la teoría estándar de la diferenciación sexual (la hipótesis activacional-organizacional).

PALABRAS CLAVE: Modelos animales; sesgo sexual; hipótesis activacional-organizacional.

\section{IT'S A MALE WORLD: THE SEXUAL BIAS OF ANIMAL MODELS IN BIOLOGY}

Copyright: (C) 2019 CSIC. Este es un artículo de acceso abierto distribuido bajo los términos de la licencia de uso y distribución Creative Commons Reconocimiento 4.0 Internacional (CC BY 4.0).

ABSTRACT: In this work we will analyze from a metatheoretical viewpoint animal models and their use in research within the life sciences. Beginning with the evaluation of a number of scientific works denouncing a sex bias in research practices using such models, we search for the implicit theoretical assumptions which provide its foundations and argue that, far from being a methodological mistake, they are part of an extensive tradition ubiquitous in the history of biology. On the other hand, we expose the theoretical motives for replacing those assumptions, by taking into account the historical and philosophical analyses of the sex differentiation' standard theory (the Organizational-Activational Hypothesis).

KEYWORDS: Animal models; sex bias; OrganizationalActivational Hypothesis. 


\section{INTRODUCCIÓN}

Los modelos animales utilizados en distintas áreas de las ciencias biológicas (como la fisiología, la farmacología y las neurociencias, entre otras) poseen por fundamento una analogía entre el sistema objetivo (en general alguna dimensión particular de la humanidad, ya sea física o conductual) y el modelo, el cual es, para estos casos un animal no-humano. Intuitivamente puede entenderse que los animales utilizados como modelo poseen como ventaja el tener similitudes con los seres humanos en múltiples aspectos, dado que, como sugiere Evelyn Fox Keller "[...] a diferencia de los modelos mecánicos y matemáticos (y este puede ser el punto crucial), los organismos modelos son ejemplares o modelos naturales -no construidos artefactualmente sino seleccionados del taller propio de la naturaleza" (Keller, 2002, p. 51). Esto se refuerza si se toma en cuenta una concepción que suponga que la diferencia entre animales y humanos sea de grados y no cualitativa (Velayos Castelo, 2013).

Aquí cabe preguntarse qué elementos propios de los animales utilizados como modelos para la investigación biológica los hace representativos en forma relevante del sistema objetivo, es decir, de algún aspecto de la biología humana o de otra especie. Una respuesta obvia pero trivial es sostener que los modelos animales poseen propiedades relevantes similares a las de los humanos dado que éstos poseen el mismo origen en "el taller propio de la naturaleza", pero esto no basta. Las homologías entre especies, en sí mismas, no agotan el problema de la representación, es decir, los modelos animales posibles están subdeterminados por las semejanzas entre especies. Ignorar dicha subdeterminación (y por ende a la plétora de opciones que existen y son teóricamente aceptables para formar modelos animales rescatando diferentes homologías) y justificar la modelización solamente en el origen común de los seres vivos es caer en un razonamiento circular que posee la forma siguiente " $A$ representa $B$ si y solo si es similar en aquellos casos en los que A representa B" (Suárez, 2003, p. 235).

Para salir de este problema entonces autores como Giere (2010) introducen elementos pragmáticos en la representación. Así, la relación aparece triádica: hay un sistema objetivo, un modelo, y un agente que intencionalmente direcciona los elementos del modelo hacia el objetivo. Así, es el agente intencional quien especifica lo que el modelo pretende representar. Lombardi, Acorinti y Martínez critican la postura pragmatista pues "[...] la defensa de tesis realistas que toda interpretación representacionalista pareciera conllevar se diluye en la voluntad del agente que proyecta y explica, pero no representa" (Lombardi et al., 2016, p. 158). Sin embargo, no parece posible prescindir legítimamente del elemento pragmático pues, como veremos en este trabajo, es este el que permite entender cómo ciertos marcos conceptuales son preferidos frente a otros, incluso cuando existe una pérdida epistémica.

Lo que discutiremos en el presente trabajo es un caso de este tipo: existe en la investigación biológica con modelos animales una tendencia profundamente marcada a utilizar animales machos en detrimento de las hembras (Wald y Wu, 2010). El segundo apartado de este trabajo será dedicado a la descripción de múltiples casos documentados de esta práctica y sus consecuencias concretas en los ámbitos científico y social. El tercer apartado explorará los motivos en los que se sostiene esta práctica, los cuales incluyen cuestiones de orden metodológico, aunque se afirmará que también incluyen supuestos teóricos implícitos pero ubicuos. El cuarto apartado partirá de un análisis filosófico de la teoría estándar de la diferenciación sexual denominada hipótesis organizacional-activacional (Phoenix, Goy, Gerall y Young, 1959) para sostener que los mencionados supuestos teóricos implícitos son cuestionables, y en el último apartado se plantearán las conclusiones finales.

\section{DESCRIPCIÓN DE LA EXCLUSIÓN DE HEMBRAS}

Aun en el sentido representacional pragmático apenas presentado, "modelos animales" se dice de muchas formas. Entre los modelos animales, Rose y Abi-Rached (2013) sostienen que es plausible distinguir: modelos de estructura, función y enfermedad, modelos conductuales y modelos de enfermedades neuropsiquiátricas. Esta distinción es importante para situar los distintos desafíos a los que se enfrentan los modelos animales. En el caso de los modelos animales de enfermedades neuropsiquiátricas, los mismos han sido objeto de fuertes críticas referidas a la traducción de la conducta deambulatoria del modelo a la sintomatología psiquiátrica de la esquizofrenia (Cynowiec, 2017) por ejemplo. Sobre los modelos animales conductuales, fundamentales en la práctica neurocientífica, Sullivan (2009) ha presentado objeciones de orden general al defender que las dos condiciones de la experimentación (fiabilidad-generalidad o replicabilidad-validez) se encuentran en contradicción en el caso de esta modelización. Dichas discusiones son centrales en el abordaje filosófico (y teórico) del uso de modelos animales. Asumiremos que, pese a la caracterización de Sullivan sobre la experimentación 
con animales en general, la modelización con animales es una práctica científica válida, en la línea de Atanasova (2015). Sea como fuere, incluso si la modelización animal no puede validarse de forma global desde la perspectiva epistemológica, no deja de ser una de las prácticas características de las ciencias de la vida contemporáneas y es por ello justamente objeto de interés de la filosofía de la ciencia.

El objetivo de este trabajo es arrojar luz desde la metateoría a una discusión que se viene dando desde al menos una década a esta parte. Diferentes autores han denunciado un sesgo o bias en el diseño de modelos animales presente en los tres subtipos planteados por Rose y Abi Rached (2013): la exclusión de las hembras. Hemos encontrado al menos una veintena de artículos, capítulos y revisiones (reviews) en los que se enfatiza, explica y cuestiona dicho sesgo. Los más completos y claros son los trabajos de Wald y Wu en Science (2010), de Beery y Zucker (2011), y los más extensos de Wizemann y Pardue (2001) y de Wizemann (2012). Ahora bien, aun cuando nos refiramos con exclusividad al sesgo en el marco de los modelos animales, no puede dejarse de lado que estas denuncias fueron largamente precedidas por el trabajo de la crítica feminista de la ciencia y de la epistemología con perspectiva de género, que denunció el androcentrismo en las ciencias biomédicas y biológicas. Nuestro propio trabajo es resultado de la apertura conceptual que las académicas feministas operaron desde los años 80 y que supuso una revisión profunda de los consensos construidos en el marco teórico y metateórico.

No obstante, hay que tener en cuenta que el androcentrismo (o sesgo masculino) se dice al menos de dos maneras. En los trabajos ya clásicos de Longino (1997), luego retomados en el monumental trabajo de Harding (1986), el sesgo masculino tiene que ver con el modo en el que ciertos prejuicios acerca de los roles de género permean la actividad científica y ponen en jaque el ethos de la objetividad y neutralidad valorativa. En este sentido, las explicaciones evolucionistas, por ejemplo, interpretan la evidencia arqueológica como confirmando la división sexual del trabajo típica de las sociedades contemporáneas (o en el caso de Darwin, de la Inglaterra victoriana) aun cuando la misma evidencia es compatible (e incluso más compatible) con explicaciones que violentan tal división. Este sesgo masculino está ampliamente extendido en la literatura feminista y en muchos casos se ha constituido en el florete conceptual con el cual se han presentado fuertes casos contra las explicaciones de la conducta humana basadas en la constitución biológi- ca de los seres humanos, siendo el ejemplar clásico la neuroendocrinología del comportamiento (véanse por ejemplo Fine 2010; van den Wijngaard, 1997).

Aun cuando el sentido apenas presentado ha supuesto una sacudida importante en el mundo de la ciencia y de la filosofía de la ciencia (ya sea de forma directa o indirecta, véase Keller, 2004), de cara a los objetivos del presente trabajo tiene una relevancia mayor el segundo sentido de androcentrismo que, aun vinculado con el primero, puede ser distinguido. En el contexto de la revisión crítica de las prácticas biomédicas, se ha denunciado repetidamente que las mujeres son excluidas del objeto de la medicina que se centra en el hombre. Desde 1980, con Alice Through the Microscope del Brighton Women an Science Group, se ha consolidado una medicina con perspectiva de género (Gender Medicine) que ha puesto el foco en el profundo desconocimiento sobre la biología específica de las mujeres, así como en el fuerte sesgo hacia lo masculino que se ha manifestado en este terreno. En palabras de Ineke Klinge:

"Desde los ochenta, los amplios esfuerzos por parte de los investigadores de género y de salud femenina en varios lugares han trabajado para "corregir" las brechas y sesgos observados. Estos esfuerzos juntos comparten el objetivo de lo que hoy ha sido reunido bajo el nombre de medicina con perspectiva de género. La medicina con perspectiva de género como una innovación de la investigación biomédica se enfoca en el sexo, el género y la salud, y aborda las problemáticas de salud de mujeres y hombres en la investigación y la práctica clínica. El objetivo de este nuevo campo de investigación es desarrollar una práctica de investigación sensible al sexo y el género, que conducirá a un conocimiento de base adecuado para el tratamiento clínico y para políticas de salud informadas. Se ha desarrollado como una vasta área de investigación, un campo de interés reconocido y de relevancia para un número de investigadores, clínicos y hacedores de políticas cada vez mayor" (Klinge y Wiesemann, 2010, p. 17).

Parece bastante obvio en qué sentido este segundo sentido de androcentrismo es más relevante para nuestro trabajo. No se trata de una mera crítica al biologicismo de ciertos enfoques, sino del reconocimiento del hecho diferencial de la mujer en el plano biológico y la necesidad urgente de otorgarle su propio lugar en tanto $50 \%$ de la especie. Nuestro trabajo puede leerse como una extensión de esta perspectiva crítica hacia el modelado con animales no humanos en el marco de las ciencias biológicas y biomédicas ${ }^{1}$. 
Volviendo a los animales no humanos, en términos cuantitativos, el sesgo es sólido y se manifiesta en las diversas subdisciplinas de la biología, con menores pero significativas excepciones. Aquí nos basaremos principalmente en los resultados del meta-análisis llevado adelante en 2009 (Beery y Zucker, 2011), el cual supone una corroboración de la investigación llevada a cabo por el grupo de trabajo de Wizemann en el 2001. Los campos disciplinares abordados fueron biología general, inmunología, neurociencia, fisiología, farmacología, reproducción, endocrinología, fisiología del comportamiento, conducta y zoología. En ocho de los diez campos analizados por Beery y Zucker se presenta con claridad el sesgo en cuestión. En neurociencias por cada artículo que utiliza exclusivamente hembras, hay 5.5 que se refieren exclusivamente a machos. Dicha proporción es también significativa en farmacología y en fisiología (5:1 y 3,7:1 respectivamente). El sesgo menos pronunciado se da en modelos conductuales (1,4:1). Por otro lado, entre el $22 \%$ y el $42 \%$ de los artículos en neurociencia, fisiología y biología general ni siquiera indican el sexo de los animales utilizados como modelo, mientras que esa cifra se eleva al $60 \%$ en el caso de la inmunología.

Lo primero que llama la atención es que el sesgo se presenta de dos modos, que si bien se encuentran íntimamente relacionados no son idénticos: por un lado, en vastas áreas de la práctica modelística, se utilizan casi en su totalidad animales macho y, por el otro, en igualmente vastas áreas no se informa sobre el sexo de los animales utilizados. Podría parecer forzado sostener, como lo hacen quienes han discutido el sesgo desde la perspectiva científica (cf. McCarthy, Arnold, Ball, Blaustein y de Vries, 2012), que ambos fenómenos (exclusión y omisión) son parte del mismo problema. $Y$ esto porque, al no indicar el sexo de los animales utilizados para el modelo, no es posible prima facie determinar si se incluyeron hembras o no, por lo cual no es claro que dicha práctica sea un caso del sesgo que nos ocupa. Independientemente de si ulteriores investigaciones puedan determinar la exclusión o no de hembras, consideramos que es válido presentarlo como un caso de la exclusión en tanto la idea que subyace a dicha omisión es que, para los fines de la modelización animal, el sexo es irrelevante (o como mínimo, lo suficientemente irrelevante como para excluirlo de la sección Materials y Methods). La idea de que el sexo no es una variable relevante en el diseño de modelos animales subyace además a la exclusión de las hembras cuando esta es mencionada, en tanto se extrapolan los resultados no solo de machos a machos de distintas especies, sino al conjunto de la especie target independientemente del sexo.
En segundo lugar, es preciso notar como, entre las disciplinas estudiadas por Beery y Zucker (2011), dos de ellas presentan un sesgo inverso al hasta ahora comentado: inmunología y reproducción. En el caso de la inmunología el poco significativo sesgo inverso parece condecirse con la ya mencionada inmensa tasa de no mención. En el caso de la reproducción, sin embargo, la omisión del sexo es prácticamente inexistente. Consideramos que este resultado refuerza una de las ideas defendidas en nuestro trabajo (como se verá más adelante en detalle): que el sexo solo es considerado una variable cuando lo estudiado afecta a las estructuras y a las funciones reproductivas, mientras que en el resto de la biología es (con altibajos pero de modo constante) considerada irrelevante.

\section{NO ES SOLO METODOLOGÍA: INTRODUCCIÓN AL SUPUESTO ONE-SEX}

La pregunta que surge a partir de lo anterior es: ¿por qué se asume que el sexo es irrelevante para la mayoría de los aspectos de la modelización? Basándonos en la literatura científica citada anteriormente, encontramos que hay respuestas de tres tipos, presentadas a continuación por orden ascendente de interés epistemológico:

Económicas, presupuestarias (externas): ciertas especies, como las wistar (macho) se han convertido en canónicas para la modelización, particularmente en neurobiología y en endocrinología del comportamiento. Una de las razones de esta elección es externa: son baratas, tienen numerosas camadas y su longevidad (lifespan) es apta para la experimentación animal. Notablemente el costo económico de las wistar macho es sensiblemente inferior al de las hembras. En términos presupuestarios, entonces, se pretende justificar el sesgo atendiendo a la optimización de recursos.

Metodológicas: siguiendo con el ejemplo de las wistar, una de las razones aducidas para la exclusión de hembras es el ciclo hormonal, considerado generalmente como un factor perturbador. Obviamente, no es que no puedan controlarse y aislarse los efectos del mismo sino que para ello es necesario elevar el número muestral de las wistar. La versión estándar de este argumento se presenta con las razones económicas, dado que aumentar el $n$ muestral implica aumentar los costos, máxime cuando las hembras son más caras que los machos.

Las ratas y ratones macho se han transformado en el modelo animal por defecto para muchas enfermedades porque son más baratos y sencillos para tra- 
bajar que las hembras. Los roedores hembra tienen un ciclo ovárico de cuatro días, por lo que los investigadores que los utilizan deben tomar diariamente hisopados vaginales en los experimentos donde las hormonas pueden jugar un rol. "De otro modo, los datos no son interpretables", dice el investigador sobre obesidad Andrew Greenberg del Human Nutrition Research Center on Aging de la Tufts University en Boston. Los científicos pueden necesitar también mantener tanto como cuatro veces el número de animales hembra sobre animales macho para asegurarse de que sus sujetos estén ciclando en sincronía. $\mathrm{E}$ incluso con esas precauciones, el ciclo puede todavía llevar a resultados menos claros que son más difíciles de publicar (Wald y Wu, 2010, p. 1571).

Conceptuales: autores como McCarthy et al. (2012) y Zuk (2002) han apuntado hacia razones de tipo conceptual para la exclusión de hembras. La difusa idea que subyace a la exclusión de las hembras es, según dichos autores, que los machos son representativos de la especie, la forma estándar o propia de la especie: "el paradigma en ciencia ha sido ver al macho de una especie, incluyendo a los humanos, como la norma, y a las hembras o mujeres como variaciones, casos especiales, excepciones a la regla" (Zuk, 2002, p. 29).

Nuestro argumento central es que, si bien las razones externas y metodológicas juegan un rol importante en la explicación del sesgo, no son condiciones suficientes (individual o conjuntamente consideradas) de la exclusión del sexo como variable relevante. Para que la práctica modelística sesgada tenga sentido, en tanto los resultados obtenidos a partir de los machos se extrapolan al conjunto de la especie independientemente de su sexo, es necesario que una razón conceptual acompañe dicha decisión. Es necesario, argumentamos, que algún presupuesto de orden teórico legitime la consideración del macho como representativo de la especie. Y nuestra aportación va en la dirección de identificar y elucidar ese presupuesto.

Ahora bien, ¿̇por qué asumir que algún tipo de teoría implícita, folk theory o presupuesto teórico está en la base de la decisión metodológica de utilizar modelos animales sesgados? Tanto McCarthy et al. y Zuk, que sostienen que hay una razón conceptual, como nosotros, que sostenemos que dicha razón conceptual descansa sobre algún presupuesto general, estamos descartando de entrada la posibilidad de que el sesgo sea nada más que un mero error, una omisión alevosa de una variable importante ${ }^{2}$. Esgrimimos dos argumentos para sostener que la exclusión de hembras no es un mero error recurrente:
En primer lugar, la modelización animal, en tanto práctica fundamental de ciertas áreas de la biología, descansa sobre la aceptación de ciertas teorías que legitiman la idea de producir conocimiento sobre rasgos de algunas especies $X$ estudiando rasgos equivalentes (en algún sentido relevante) de otras especies Y. Así mismo, la relación entre teorías y práctica modelística es bidireccional, como señala Hedges: la elección de ciertos organismos como modelos de otros presupone ciertas teorías (genética, biología evolutiva) pero además:

"Los organismos modelo representan solo una pequeña fracción de la biodiversidad que existe en la tierra, aunque la investigación que ha resultado de su estudio forma el núcleo del conocimiento biológico. Históricamente, las comunidades de investigación [...] se han enfocado en estos organismos modelo para obtener una visión dentro de los principios generales que subyacen a varias disciplinas, como la genética, el desarrollo y la evolución" (Hedges, 2002, p. 838).

Puede parecer trivial sostener que metodología y teoría tienen algún grado de imbricación. La modelización animal tiene sentido si y solo si la especie target y el modelo son similares en algún aspecto relevante y esa similitud descansa sobre las bases epistémicas de la genética, de la evolución y del desarrollo (entre otras). Son las propiedades únicas del genoma del pez globo y las macroneuronas en el caso de las medusas las que hacen a dichas especies organismos interesantes para su utilización modelística, y no su disponibilidad masiva o su precio de mercado. Ahora bien, si aceptamos que son las teorías generalmente aceptadas las que dan razón a las prácticas modelísticas, es decir, que son dichas teorías la que legitiman la homología entre especies, entonces debemos aceptar mutatis mutandis que algún presupuesto de orden teórico legitima el uso de animales macho como modelo de una especie. En otras palabras, si es cierto que es el conocimiento previo acerca de las similitudes las que nos permite decir que $X$ especie puede modelar $a$ otra especie $\mathrm{Y}$, entonces también es cierto que algún conocimiento generalmente aceptado nos permite decir que uno de los sexos (macho) es representativo del conjunto de una especie sexualmente dividida (machos y hembras).

En segundo lugar, sin tener en cuenta la perspectiva de las teorías que legitiman las prácticas experimentales y modelísticas, es decir, limitándonos al análisis del procedimiento modelístico como tal, podríamos pensar que la premisa "no hay diferencias relevantes entre machos y hembras (en las variables nómi- 
camente importantes para tal o cual modelo)" es un proviso (Hempel, 1988), es decir, una presuposición o inferencia teórica no explícita pero esencial en explicaciones o predicciones dentro de esquemas nomológico-inferenciales (véase Díez, 2002). Estas premisas que lidian con potenciales perturbaciones al sistema bajo estudio poseen un peso lógico importante dentro del razonamiento con leyes científicas y suelen no ser explicitadas debido a que el científico puede considerarlas obvias o directamente no tenerlas en cuenta. En nuestro caso, podemos plantear que, una vez explicitado, el proviso para uno de los ejemplos del sesgo se formularía como "los testeos en animales demuestran que la droga $X$ es segura para seres humanos siempre y cuando los animales macho sean suficientemente representativos tanto de humanos macho como hembra". Estructuralmente la premisa "Ios animales macho son suficientemente representativos tanto de humanos macho como de hembra" funciona, en efecto, como un proviso (en cuanto a su función dentro de las explicaciones o predicciones). No obstante, parece que la salvedad introducida por dicha premisa excede lo aceptable puesto que el sexo acarrea una serie de propiedades del modelo y de su target que son esenciales en la relación de representación, a diferencia de la temperatura alta en la teoría magnética, la cual funciona simplemente como factor perturbador. Así pues, el sexo en el marco modelístico es más bien una variable relevante que sospechosamente no es explicitada. Los trabajos científicos a los que hacen alusión Beery y Zucker (2011) no explicitan que el sexo del modelo animal sea irrelevante, simplemente operan como si así fuese.

Ahora bien, si la exclusión de las hembras no puede explicarse sin recurso a factores teóricos, pues ni es un error contingente ni es un proviso, queda identificar adecuadamente de qué tipo de presupuesto se trata. Retomando el estudio de Beery y Zucker (2011), es notable que aquellos campos donde el sesgo es menor o inexistente son los relacionados con la reproducción. Las diferencias entre machos y hembras parecen limitarse entonces a la función reproductiva. Es igualmente notorio que entre los argumentos delineados para sostener que la exclusión es fundamentalmente correcta, se insista en las dificultades derivadas del ciclo hormonal femenino, es decir, de su función reproductiva. Al menos desde Aristóteles, y bajo distintas formas a lo largo de más de 2.000 años de historia de la biología y de la filosofía de la naturaleza, se ha asumido que las diferencias entre machos y hembras tienen su sustrato en la dicotomía gonadal establecida por la función reproductiva (cf. Laqueur,
1994). Tras el desarrollo de la genética, se introduce un eje más a la diferenciación sexual, constituyéndose así la visión estándar sobre la división sexual como una cadena causal de tres elementos: cromosomas (que determinan) gónadas (que determinan) rasgos sexuales secundarios. Las diferencias sexuales se explican, entonces, con recurso al proceso de diferenciación lineal, siendo las diferencias vinculadas con la reproducción (eje gonadal) las fundamentales.

A esta concepción que coloca en el centro de las diferencias y de la diferenciación sexual a las estructuras y a la función reproductiva la llamamos gonadocéntrica. Dicha concepción, además, considera que, siendo las diferencias exclusivamente reproductivas, es el macho quien representa a la especie en su conjunto y la hembra es una desviación respecto a la media. Por tanto, la elección terminológica debida a Zuk (2002), male-model, capta adecuadamente el resultado modelístico y práctico de esta asunción gonadocéntrica. Un ejemplo que refuerza esta idea es el hecho de que esta indistinción aparente permite modelar machos y hembras (humanos o de otra especie) con ejemplares macho, pero curiosamente, no permite modelar machos y hembras con ejemplares hembra.

El paso del gonadocentrismo a los modelos animales sesgados es ahora fácil de ver. Si es cierto que las diferencias entre machos y hembras están restringidas a la función y a las estructuras reproductivas entonces, a menos que el modelo pertenezca a la biología reproductiva, los machos son representativos de la especie. Los resultados obtenidos en modelos animales exclusivamente masculinos se extrapolan a hombres y mujeres por igual, teniendo en el peor de los casos que introducir factores de seguridad (Calabrese, 1985) vinculados a la carga hormonal diferencial y al dimorfismo de altura y peso promedio.

¿Acaso es correcto desde un punto de vista teórico aceptar el presupuesto gonadocéntrico?

\section{LA TEORÍA OA: EL MOSAIQUISMO CONTRA EL SUPUESTO ONE-SEX}

El supuesto teórico gonadocéntrico, hemos defendido, subyace a la asunción de que los machos son representativos de una especie, y que las diferencias entre machos y hembras quedan circunscritas a las funciones y a las estructuras reproductivas. Este supuesto teórico explicaría los resultados arrojados por los ya mencionados estudios de Beery y Zucker (2011), Wizemann y Pardue (2001) y Wizemann (2012). Ahora bien, podría sostenerse que el supuesto aquí señala- 
do, independientemente de su grado de explicitación, no es un problema ni epistémico ni de otro tipo. En el presente apartado intentaremos mostrar que el supuesto que legitima la exclusión de las hembras en la modelización animal es al menos sospechoso respecto de las teorías vigentes sobre las diferencias y la diferenciación sexual.

El célebre paper de 1959, Organizing Action of Prenatally Administered Testosterone Propionate on the Tissues Mediating Mating Behavior in the Female Guinea Pig de Charles Phoenix, Robert Goy, Arnold Gerall y William Young es usualmente considerado el hito fundacional de la hipótesis organizacional-activacional (OA) (véase Arnold, 2009; McCarthy y Arnold, 2011). Si bien esto es discutible (como los propios autores reconocen al citar los trabajos previos de Vera Dantchakoff de 1938), lo que queda fuera de toda duda es que Organizing Action... es la primera confirmación experimental de la OA y la primera presentación relativamente sistemática y programática de la teoría, constituyendo la primera aplicación exitosa de dos ideas fundamentalmente rupturistas y programáticas respecto a las posiciones generalmente aceptadas en torno a la naturaleza de las diferencias y de la diferenciación sexual.

En primer lugar, el rol de las hormonas en la regulación (activación/inhibición) de conductas sexualmente dimórficas era algo bien conocido en el año 59. De hecho, uno de los firmantes del artículo era Young, considerado generalmente el fundador, junto (pero independientemente) con Frank Beach (Beach y Holz, 1946; Beach, Noble y Orndoff, 1969; Young, Dempsey y Myers, 1935), de la endocrinología del comportamiento. En efecto, desde los años 30, Young había mostrado el rol crucial que el estradiol y la progesterona juegan en la activación de las conductas reproductivas en las hembras. Los resultados de 1959, no obstante, dieron apoyo a la idea que otorgaba a la acción hormonal un rol mucho más fundamental en la explicación de las conductas sexualmente dimórficas: cuando ocurren en períodos sensibles del desarrollo embrionario, las hormonas producen efectos organizacionales, es decir, permanentes cambios en las estructuras y funciones neurales responsables de las conductas sexuales. El ejemplar paradigmático de este proceso es la reversión completa o parcial de las conductas reproductivas en roedores (monta en machos y lordosis en hembras) (Houtsmuller et al., 1994).

Ahora bien, los resultados del trabajo de Phoenix et al. (1959) sugieren no solo este rol dual de la acción hormonal en la diferenciación sexual sino que también indican que el soma o substrato sobre el que las hormonas actúan es el sistema nervioso central. Al menos desde Aristóteles y su Reproducción de los animales (Sánchez, 1994), el eje sobre el que se asientan las diferencias sexuales era el gonadal. Dado que machos y hembras existen como resultado de la necesidad de perpetuar la especie, y ser macho o hembra significa ser capaz de cumplir cierta función en el marco reproductivo, entonces las diferencias entre machos y hembras están determinadas por las estructuras que permiten la ejecución de dicha función. Obviamente el desarrollo de la biología moderna, con particular énfasis en la endocrinología, permite una sofisticación del principio gonadal de la diferenciación: efectivamente las gónadas tienen un rol fundamental en las diferencias entre machos y hembras porque son las productoras de hormonas, fundamentales a su vez en el proceso de diferenciación, particularmente en la madurez sexual. Posiciones explícitamente partidarias del gonadocentrismo pueden encontrarse hasta la década de los setenta del siglo pasado. Frank Beach, en franca polémica con los proponentes de la OA, sostuvo durante toda su carrera que la acción organizacional y activacional de las hormonas en la diferenciación sexual tiene como sustrato el aparato reproductor y no el sistema nervioso central y no aceptó hasta 1975 que, al menos en determinados casos, las hormonas pueden producir cambios permanentes en las estructuras neurofisiológicas.

Informalmente la ley fundamental de la OA puede presentarse como sigue:

Para toda conducta dimórfica en la especie, existen hormonas que durante el período organizacional determinan estructuras neurofisiológicas dimórficas y durante el periodo activacional determinan dichas conductas.

Ahora bien, como acabamos de mostrar, la OA es la teoría estándar para la diferenciación conductual en mamíferos. De hecho, es notable que una de las subdisciplinas donde el sesgo es menor de acuerdo con Beery y Zucker (2011) es la de la biología del comportamiento. Podría por tanto decirse en una primera instancia que el sesgo no contraviene ninguna de las teorías generalmente aceptadas, pues donde se ha mostrado suficientemente la relevancia de dichas diferencias es en el apartado conductual, permaneciendo lo demás inalterado respecto de la asunción gonadocéntrica.

Independientemente de si el explanandum de la OA se circunscribe al campo de las conductas dimór- 
ficas, una de las consecuencias más interesantes de su aplicación en tanto programa científico es la búsqueda sistemática de estructuras neurofisiológicas que se corresponden con las conductas objeto de estudio (una buena síntesis de los resultados de dicha búsqueda puede encontrarse en Simerly, 2002). Así mismo, y como se indica en la mayoría de los manuales de neurociencia (Fink, Pfaf y Levine, 2011), las diferencias halladas en el camino desde la OA exceden el plano fisiológico, pudiéndose encontrar diferencias notables en la neuroquímica, en la sensibilidad a ciertas enfermedades, etc. La OA, por tanto, constituye el primer capítulo de una ciencia de las diferencias sexuales y del proceso de diferenciación sexual, que cruza transdisciplinariamente la genética, la endocrinología, la neurociencia y la endocrinología.

Blanchard, Griebel y Blanchard (1995) muestran cómo los estudios preclínicos con modelos animales en el campo de la ansiedad y el pánico (enfermedades que afectan notablemente más a mujeres que a hombres) únicamente utilizan machos, dando lugar a un conocimiento superficial sobre los mecanismos neurocomportamentales de esta enfermedad. Pero lo más peligroso es la incidencia del sesgo en las pruebas de fármacos:

"[...] las curvas dosis-respuesta para mujeres pueden ser diferentes de aquellas para hombres, o incluso puede ser que algunas drogas sean más útiles o apropiadas para sujetos de un género que para el otro. Si el efecto de un compuesto es afectado por (por ejemplo) las características hormonales del sujeto, esta información podría ser utilizada para mejorar el tratamiento tanto en hombres como mujeres, pero es más probable que tenga un impacto mayor en el segundo caso" (Blanchard et al., 1995, p. 81).

Por otro lado, de las diez drogas retiradas del mercado por parte de la Food and Drug Administration (FDA) de Estados Unidos en el año 2001, siete de ellas presentaron evidencia de poseer un riesgo mayor para mujeres que para hombres (Drug Safety: Most Drugs Withdrawn in Recent Years Had Greater Health Risks for Women), entre las que se encontraban los supresores de apetito Pondimin y Redux, los antihistamínicos Seldane e Hismanal, la droga cardiovascular Posicor, el medicamento para la diabetes Rezulin y los fármacos para el tracto gastrointestinal Propulsid y Lotronex. La neurocientífica de la Columbia University Rae Silver ha planteado que el motivo de la introducción de estos fármacos peligrosos en el mercado se debió al sesgo en los estudios preclínicos a partir de modelos animales (Wald y Wu, 2010).
En definitiva, parece que existe, por un lado, una teoría generalmente aceptada (mainstream) que invalida la modelización sexualmente sesgada y, por el otro, un presupuesto teórico que establece la representatividad de los machos sobre el total de la especie (modelo y modelada). El hecho de que esta teoría aceptada no sea considerada en la práctica modelística parece sostenerse únicamente en decisiones de corte pragmático como las que según Giere (2010) sostienen a la tríada que da fundamento a la modelización (sistema objetivo, modelo, agente) al direccionar, como mencionamos en la primera sección, los elementos del modelo hacia el sistema objetivo. Si bien no es materia de este trabajo profundizar en ello, dichos elementos pragmáticos parecen vincularse con las razones externas y metodológicas que señalábamos en ese apartado, y presumiblemente con otros componentes volicionales de corte ideológico.

Todo lo argumentado hasta aquí hace razonable y precautorio la defensa de un cambio en el supuesto teórico que subyace al sesgo. Dicho cambio implica, por supuesto, la aceptación de una perspectiva donde el sexo es mucho más relevante de lo que se sostiene hasta ahora. Las hembras deben tener su lugar como objeto de la investigación científica, no solo por razones epistémicas, sino también para evitar los mencionados resultados dañinos en el ámbito social que se han dado como producto de dicha exclusión.

\section{BALANCE Y PERSPECTIVAS}

El presente trabajo constituye la primera aproximación filosófica a la problemática del sesgo sexual en modelos animales. Hemos mostrado suficientemente que el sesgo tiene una dimensión teórica que no puede ser escamoteada con recurso a cuestiones enteramente metodológicas y económicas. Hemos defendido además que el presupuesto general que subyace a la exclusión de las hembras en el diseño de modelos animales es gonadocéntrico y sesgado, y que contraviene a la visión mosaico y compleja que la contemporánea biología sostiene respecto de la diferenciación sexual. En este sentido, hemos presentado sucintamente la OA y el programa científico resultante como contraejemplo flagrante del principio gonadocéntrico y del sesgo.

Creemos que la labor filosófica sobre la ciencia no solo responde a intereses intrínsecos a la disciplina sino que puede realizar aportaciones relevantes a la actividad científica y sus resultados, no siempre estrictamente epistémicos. En este sentido, el sesgo aquí analizado se ha mostrado no solo sospechoso en términos cognoscitivos, sino también en términos 
sociales: algunas de las drogas retiradas del mercado estadounidense por su riesgo incrementado en mujeres, como por ejemplo el antihistamínico Seldane, han sido causa de muertes (al menos ocho, según el New York Times, 1997, 17 de enero).

Obviamente hemos dejado para más adelante sustantivos problemas metateóricos que la cuestión suscita. Principalmente nuestra caracterización del gonadocentrismo demanda una revisión de las historias de la diferenciación sexual hoy en día canónicas, primordialmente debidas a Thomas Laqueur (1994). De acuerdo con Laqueur, la aparición de un marco conceptual que concibe a machos y hembras como constitutivamente diferentes nace al final de la ilustración, reemplazando al one-sex-model, que establece que las hembras son versiones imperfectas de los machos, y que habría caracterizado al pensamiento clásico sobre lo vivo, con particular importancia en Aristóteles y Galeno (Connel, 2000). Lo que aquí estamos afirmando se contrapone con lo anterior en que las investigaciones en ciencias de la vida a partir de modelos animales parecen perpetuar el one-sexmodel pre-ilustración, en contraposición al two-sexmodel moderno sugerido por Laqueur, cuya postura en consecuencia debiera ser matizada.

Finalmente, una perspectiva analítica que se abre es la de entender en qué sentido preciso se vinculan el androcentrismo tal y como es denunciado en la tradición epistemológica feminista con el sesgo en los modelos animales.

\section{AGRADECIMIENTOS}

Los autores agradecen al doctor Santiago Ginnobili sus valiosas sugerencias tras una lectura atenta del manuscrito y a un evaluador anónimo sus cruciales observaciones. La investigación se ha llevado a cabo en el seno los proyectos PICT-2014-1741, PICT-2012-2662 (ANPCyT, Argentina), PIP 112-201101-01135 (CONICET, Argentina) y 32/15 255 (UNTREF, Argentina).

\section{NOTAS}

1. Un estudio exhaustivo y en el que ambos sentidos de androcentrismo son relacionados puede encontrarse en Mujer, salud y poder de Carmen Valls-Llobet (2009).
2. En relación a lo que venimos argumentando hasta aquí, hay una premisa implícita: que el sesgo es efectivamente un problema epistémico o metodológico. Eso, por supuesto, debe ser argumentado en función de las teorías científicas disponibles. Nosotros discutimos esta cuestión en el apartado inmediatamente posterior.

\section{BIBLIOGRAFÍA}

Arnold, A. P. (2009). The OrganizationalActivational Hypothesis as the Foundation for a Unified Theory of Sexual Differentiation of all Mammalian Tissues. Hormones and Behavior, 55 (5), pp. 570-578. https://doi.org/10.1016/j. yhbeh.2009.03.011

Atanasova, N. (2015). Validating Animal Models. Theoria, 30 (2), pp. 163-181. https://doi.org/10.1387/theoria.12761

Beach, F. A. (1975). Hormonal Modification of Sexually Dimorphic Behavior. Psychoneuroendocrinology, 1 (1), pp. 3-23. https://doi.org/10.1016/03064530(75)90019-0

Beach, F. A. y Holz, A. M. (1946). Mating Behavior in Male Rats Castrated at Various Ages and Injected with Androgen. Journal of Experimental Zoology, 101 (1), pp. 91-142. https://doi.org/10.1002/ jez.1401010107

Beach, F. A., Noble, R. G. y Orndoff, R. K. (1969). Effects of Perinatal Androgen
Treatment on Responses of Male Rats to Gonadal Hormones in Adulthood. Journal of Comparative and Physiological Psychology, 68 (4), pp. 490-497. https://doi.org/10.1037/h0027658

Beery, A. K. y Zucker, I. (2011). Sex Bias in Neuroscience and Biomedical Research. Neuroscience and Biobehavioral Reviews, 35 (3), pp. 565-572. https://doi. org/10.1016/j.neubiorev.2010.07.002

Birke, L., Faulkner, W., Janson-Somith, D. y Overfield, K. (eds.) (1980). Alice through the microscope: the power of science over women's lives. London: Virago.

Blanchard, C. D., Griebel G. y Blanchard R. J. (1995). Gender Bias in the Preclinical Psychopharmacology of Anxiety: Male Models for (Predominantly) Female Disorders. Journal of Psychopharmacology, 9 (2), pp. 79-82. https://doi. org/10.1177/026988119500900201

Calabrese, E. (1985). Uncertainty Factors and Interindividual Variation. Regu- latory Toxicology and Pharmacology. 5 (2), pp. 190-196. https://doi. org/10.1016/0273-2300(85)90032-7

Connell, S. (2000). Aristotle and Galen on Sex Difference and Reproduction: a New Approach to an Ancient Rivalry. Studies in History and Philosophy of Science, 31 (3), pp. 405-427. https://doi. org/10.1016/S1369-8486(00)00007-8

Cynowiec E. (2017). Análisis epistemológico de la construcción y utilización de modelos animales para el estudio de alteraciones psiquiátricas humanas. [Tesis doctoral inédita]. Buenos Aires: Universidad de Buenos Aires.

Danchakoff, V. (1938). La folliculine dans l'histogenèse sexuelle de l'embryon des Mammifères. Comptes Rendus de l'Académie des Sciences, 206, pp. 945-947.

Díez, J. A. (2002). Explicación, unificación y subsunción. En González, W. J. (ed.). Pluralidad de la explicación científica. Barcelona: Ariel, pp. 73-93. 
Fine, C. (2010). Delusions of Gender: How Our Minds, Society, and Neurosexism Create Difference. New York: W. W. Norton.

Fink, G., Pfaf, D. y Levine, J. (2011). Handbook of Neuroendocrinology. Oxford: Academic Press.

Giere, R. N. (2010). An Agent-Based Conception of Models and Scientific Representation. Synthese. 172 (2), pp. 269281. https://doi.org/10.1007/s11229009-9506-z

Harding, S. (1986). The Science Question in Feminism. New York: Cornell University.

Hedges, S. (2002). The Origin and Evolution of Model Organism. Nature Reviews Genetics, 3 (11), pp. 838-849. https://doi. org/10.1038/nrg929

Hempel, C. G. (1988). Provisoes: A Problem Concerning the Inferential Function of Scientific Theories. Erkenntnis. 28 (2), pp. 147-164. https://doi.org/10.1007/ BF00166441

Houtsmuller E. J., Brand, T., de Jonge F. H., Joosten, R. N., van de Poll, N. E. y Slob, A. K. (1994). SDN-POA Volume, Sexual Behavior, and Partner Preference of Male Rats Affected by Perinatal Treatment with ATD. Physiology \& Behavior, 56 (3), pp. 535-541. https://doi. org/10.1016/0031-9384(94)90298-4

Keller, E. F. (2002). Making Sense of Life: Explaining Biological Development with Models, Metaphors and Machines. Cambridge: Harvard University Press.

Keller, E. F. (2004). What impact, if any, has feminism had on science. Journal of Bioscience, 29 (1), pp. 7-13. https://doi. org/10.1007/BF02702556

Klinge, I. y Wiesemann, C. (eds.) (2010). Sex and Gender in Biomedicine: Theories, Methodologies, Results. Göttinger Universitätsverlag. https://doi.org/10.17875/ gup2010-394

Laqueur, T. (1994). La construcción del sexo: cuerpo y género desde los griegos hasta Freud. Madrid: Cátedra.
Lombardi, O., Acorinti, H. y Martínez, J. C. (2016). Modelos científicos: el problema de la representación. Scientiae Studia, 14 (1), pp. 151-174.

Longino, H. E. (1997). Feminismo y filosofía de la ciencia. En: González, M. I., López Cerezo, J. A. y Luján, J. L. Ciencia, tecnología y sociedad. Barcelona: Ariel, pp. 71-83.

McCarthy, M. M. y Arnold, A. P. (2011). Reframing Sexual Differentiation of the Brain. Nature Neuroscience, 14 (6) pp. 677-683. https://doi.org/10.1038/ nn.2834

McCarthy, M. M., Arnold, A. P., Ball, G., Blaustein, J. y de Vries, G. (2012). Sex Differences in the Brain: the not so Inconvenient Truth. The Journal of Neuroscience, 32 (7), pp. 2241-2247. https://doi.org/10.1523/JNEUROSCl.5372-11.2012

Phoenix, C., Goy, R., Gerall, A. y Young, W. C. (1959). Organizing Action of Prenatally Administered Testosterone Propionate on the Tissues Mediating Mating Behavior in the Female Guinea Pig. Endocrinology, 65 (3), pp. 369-382. https://doi. org/10.1210/endo-65-3-369

Rose, N. y Abi-Rached, J. M. (2013). Neuro: The New Brain Sciences and the Management of the Mind. Princeton: Princeton University Press.

Sánchez, E. (ed.) (1994). Aristóteles. Reproducción de los animales. Madrid: Gredos.

Simerly, R. B. (2002). Wired for Reproduction: Organization and Development of Sexually Dimorphic Circuits in the Mammalian Forebrain. Annual Review of Neuroscience, 25 (1), pp. 507-536. https://doi.org/10.1146/annurev.neuro.25.112701.142745

Suárez, M. (2003). Scientific Representation: Against Similarity and Isomorphism. International Studies in the Philosophy of Science, 17 (3), pp. 225-244. https://doi. org/10.1080/0269859032000169442
Sullivan, J. A. (2009). The Multiplicity of Experimental Protocols: A Challenge to Reductionist and Non-Reductionist Models of the Unity of Neuroscience. Synthese, 167 (3), pp. 511-539. https:// doi.org/10.1007/s11229-008-9389-4

The Hazards of Seldane (1997, 17 de enero). New York Times. [En línea]. Disponible en http://www.nytimes.com/1997/01/17/ opinion/the-hazards-of-seldane.html

Valls-Llobet, C. (2009). Mujeres, salud y poder. Madrid: Cátedra.

Velayos Castelo, C. (2013). La frontera animalhumano. Arbor, 189 (763), a065. https:// doi.org/10.3989/arbor.2013.763n5002

Wald, C. y Wu, C. (2010). Of Mice and Women: The Bias in Animal Models. Science, 327, pp. 1571-1572. https://doi. org/10.1126/science.327.5973.1571

Wijngaard, M van den (1997). Reinventing the Sexes: The Biomedical Construction of Femininity and Masculinity. Bloomington: Indiana University Press.

Wizemann, T. M. (2012). Sex-Specific Reporting of Scientific Research. A Workshop Summary. Washington: National Academies Press.

Wizemann, T. M. y Pardue, M. (2001). Exploring the Biological Contributions to Human Health. Does Sex Matter? Washington: National Academies Press.

Young, W. C., Dempsey, E. W. y Myers, H. I. (1935). Cyclic Reproductive Behavior in the Female Guinea Pig. Journal of Comparative Psychology, 19 (2), pp. 313-335. https://doi.org/10.1037/h0060351

Zuk, M. (2002). Sexual Selections: What We Can and Can't Learn about Sex from Animals. Berkeley: University of California Press.

\section{Otros recursos}

Drug Safety: Most Drugs Withdrawn in Recent Years had Greater Health Risks for Women. United States General Accounting Office (January 19, 2001). [En línea]. Disponible en: https://www.gao. gov/new.items/d01286r.pdf 